\title{
Dimorfismo sexual, uso do ambiente e abundância sazonal de Elachistocleis cf. ovalis (Anura: Microhylidae) em um remanescente de Cerrado no estado de São Paulo, sudeste do Brasil
}

\author{
Maria Tereza Chiarioni Thomé $e^{1,3}$ \& Cínthia Aguirre Brasileiro ${ }^{1,2}$ \\ http://www.biotaneotropica.org.br/v7n1/pt/abstract?article+bn00307012007 \\ Recebido em 15/08/06 \\ Versão Reformulada recebida em 10/11/06 \\ Publicado em 01/01/07 \\ ${ }^{I}$ Departamento de Ecologia, Instituto de Biociências, Universidade de São Paulo, \\ Rua do Matão, Travessa 14 s/n, CEP 05508-900, São Paulo, SP, Brasil \\ ${ }^{2}$ Museu de História Natural, Instituto de Biologia, Universidade Estadual de Campinas, \\ CP 6109, CEP 13083-970, Campinas, SP, Brasil \\ ${ }^{3}$ Autor para correspondência: Maria Tereza Chiarioni Thomé, e-mail: mtcthome@yahoo.com.br
}

\begin{abstract}
Thomé, M.T.C. \& Brasileiro, C.A. Sexual Dimorphism, habitat use and seasonal abundance of Elachistocleis cf. ovalis (Anura: Microhylidae) in a Cerrado remnant of São Paulo state, southeastern Brazil. Biota Neotrop. Jan/Apr 2007 vol. 7, no. 1 http://www.biotaneotropica.org.br/v7n1/pt/abstract?article+bn00307012007 ISSN 1676-0603.

Elachistocleis ovalis is a species presenting taxonomical problems and wide distribution throughout South America. We provide information on sexual dimorphism, calling site, habitat use and seasonal abundance of E. cf. ovalis in a preserved Cerrado area in São Paulo State, southeastern Brazil. Sampling was carried out primarily with pitfall traps. Traps were placed in areas characterized by three different physiognomies: campo sujo, campo cerrado, and gallery forest edge. We found sexual dimorphism, with females being larger than males. Similar dimorphism has already been documented for other populations and congeneric species. The calling site of males was also in agreement with the literature on other populations and species. Habitat use was different among the three environments, with higher abundance in gallery forest edges. This population showed a clear pattern of seasonal activity, in which abundance was higher during rainy seasons, as well as other Cerrrado anuran species. Abundance was related to relative air humidity and maximum temperature, two variables whose influence on anurans has already been documented. We found no variation in the abundance patterns of the population between the two years in which samples were carried out.
\end{abstract}

Keywords: sexual dimorphism, calling site, habitat use, seasonal abundance, Elachistocleis ovalis, Cerrado, São Paulo,

\section{Resumo}

Thomé, M.T.C. \& Brasileiro, C.A. Dimorfismo sexual, uso do ambiente e abundância sazonal de Elachistocleis cf. ovalis (Anura: Microhylidae) em um remanescente de Cerrado no Estado de São Paulo, sudeste do Brasil. Biota Neotrop. Jan/Apr 2007 vol. 7, no. 1 http://www.biotaneotropica.org.br/v7n1/pt/abstract?article+bn00307012007 ISSN 1676-0603.

Elachistocleis ovalis é uma espécie de taxonomia controversa e de distribuição ampla na América do Sul. Apresentamos informações sobre dimorfismo sexual, sítio de vocalização, uso do ambiente e abundância sazonal de E. cf. ovalis em um ambiente de Cerrado preservado no Estado de São Paulo, sudeste do Brasil. As coletas foram realizadas principalmente com armadilhas de interceptação e queda, instaladas em três fisionomias: campo sujo, campo cerrado e borda de mata de galeria. Foi verificado dimorfismo sexual, com fêmeas maiores do que os machos, semelhante ao documentado para outras populações e espécies congenéricas. O sítio de vocalização dos machos também foi semelhante ao registrado para outras populações e espécies do gênero. O uso do hábitat foi diferenciado na população estudada, com maior abundância em borda de mata de galeria. A população apresentou padrão marcante de atividade sazonal, no qual a abundância de indivíduos foi maior nas estações chuvosas, de modo semelhante a diversas espécies de anuros de Cerrado. A abundância foi relacionada com a umidade relativa do ar e temperatura máxima, duas variáveis cuja influência sobre a atividade dos anuros já foi documentada. Não foi observada variação anual nos padrões de abundância da população entre os dois anos de estudo.

Palavras-chave: dimorfismo sexual, sítio de vocalização, uso do hábitat, sazonalidade, Elachistocleis ovalis, Cerrado, São Paulo. 


\section{Introdução}

Elachistocleis ovalis Schneider 1799 apresenta ampla distribuição na América do Sul, se estendendo do Panamá e Colômbia, pelo leste dos Andes, à Argentina e ilha de Trindade (Frost 2004). A taxonomia desta espécie é controversa e sofreu 14 alterações desde sua descrição como Rana ovalis em 1799. Elachistocleis ovalis ocorre em sintopia com outras espécies congenéricas e também taxonomicamente problemáticas (ver Kwet \& Di Bernardo 1998 e Lavilla et al. 2003), o que pode levar a identificações pouco confiáveis. Apesar do status de conservação não ser preocupante, aparentemente E. ovalis representa um complexo de espécies, o que é agravado pela localidade desconhecida do holótipo (Lavilla et al. 2003). Esta espécie, como muitas outras de distribuição ampla, carece de estudos taxonômicos representativos para que seu status de conservação seja determinado com segurança.

O modo de vida de E. ovalis é caracterizado como fossorial (Kwet \& Di-Bernardo 1999) ou semifossorial (Achaval \& Olmos 2003) e criptozóico (Strussmann 2000). A dieta é especializada, restrita a formigas e cupins (Solé et al. 2002). Quanto à reprodução, E. ovalis apresenta padrão explosivo (sensu Wells 1977, Toledo et al. 2003), semelhante ao observado para outras espécies do gênero (Kwet \& Di Bernardo 1998 e 1999; Rodrigues et al. 2003). Ovos e larvas desenvolvem-se em corpos de água lênticos (Toledo et al. 2003) e a dieta dos girinos é composta principalmente por algas perifíticas (Rossa-Feres et al. 2004).

Neste estudo são apresentadas informações sobre a biologia de Elachistocleis cf. ovalis (Figura 1) em uma área preservada de Cerrado no Estado de São Paulo, sudeste do Brasil. Foi investigada presença de dimorfismo sexual e caracterizado o sítio de vocalização dos machos. O uso do hábitat foi avaliado através da comparação da abundância em três fisionomias distintas: campo sujo, campo cerrado e borda de mata de galeria. A abundância sazonal foi analisada ao longo de dois anos para verificar a influência de variáveis climáticas na atividade da população. Devido à rápida destruição que o Cerrado vem sofrendo nas últimas décadas (Cavalcanti \& Joly 2002) estudos sobre sua fauna tornam-se cada vez mais urgentes, principalmente nos estados mais afetados como o de São Paulo (Mantovani \& Pereira 1998). Este é o primeiro estudo abordando aspectos populacionais desta espécie em área preservada no sudeste do Brasil.

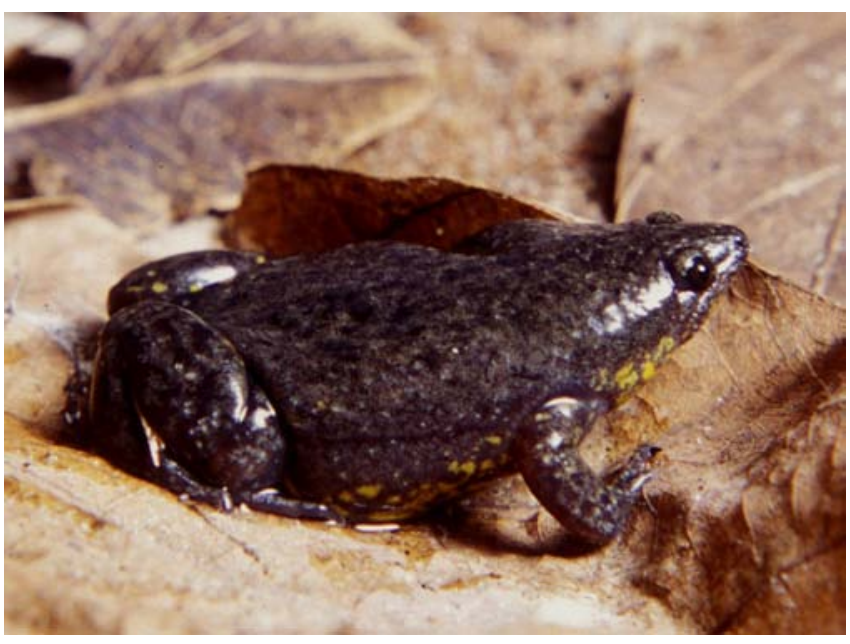

Figura 1. Elachistocleis cf. ovalis da Estação Ecológica de Itirapina.

Figure 1. Elachistocleis cf. ovalis from Estação Ecológica de Itirapina.

\section{Material e Métodos}

\section{1. Área de estudo}

O estudo foi realizado na Estação Ecológica de Itirapina (EEI) $\left(22^{\circ} 00^{\prime}\right.$ a $22^{\circ} 15^{\prime} \mathrm{S}$ e $47^{\circ} 45^{\prime}$ a $\left.48^{\circ} 00^{\prime} \mathrm{O}\right)$, um dos últimos remanescentes de Cerrado no estado de São Paulo, localizado nos municípios de Itirapina e Brotas, sudeste do Brasil (Figura 2). A região encontrase na porção Sul do domínio do Cerrado, em uma bacia sedimentar caracterizada por solos predominantemente arenosos (Vicente et al. 2005). A EEI é uma unidade de conservação estadual com 2430 ha de Cerrado preservado, constituída principalmente por campos e matas de galeria (Brasileiro et al. 2005). Este estudo foi realizado principalmente nas fisionomias de campo sujo, campo cerrado e borda de mata de galeria.

O clima na região é mesotérmico com sazonalidade acentuada (Brasileiro et al. 2005), podendo ser tipicamente reconhecida uma estação seca bem pronunciada de temperaturas mais amenas, de abril a setembro, e uma estação chuvosa de outubro a março, com temperaturas e pluviosidade mais elevadas. De julho de 2000 a junho de 2005, as médias mensais de temperatura máxima atingiram $34,5^{\circ} \mathrm{C}$ durante as estações chuvosas, enquanto que o menor valor de temperatura mínima média foi $7,9^{\circ} \mathrm{C}$, registrado durante a estação seca. A pluviosidade total mensal atingiu $433 \mathrm{~mm}$ durante a estação chuvosa, sendo que durante as estações secas houve meses sem qualquer registro de chuva.

\section{Coleta de dados}

As amostragens foram realizadas em dois períodos distintos: um período de coletas preliminares e um segundo de amostragens regulares. O período de coletas preliminares foi de setembro de 1999

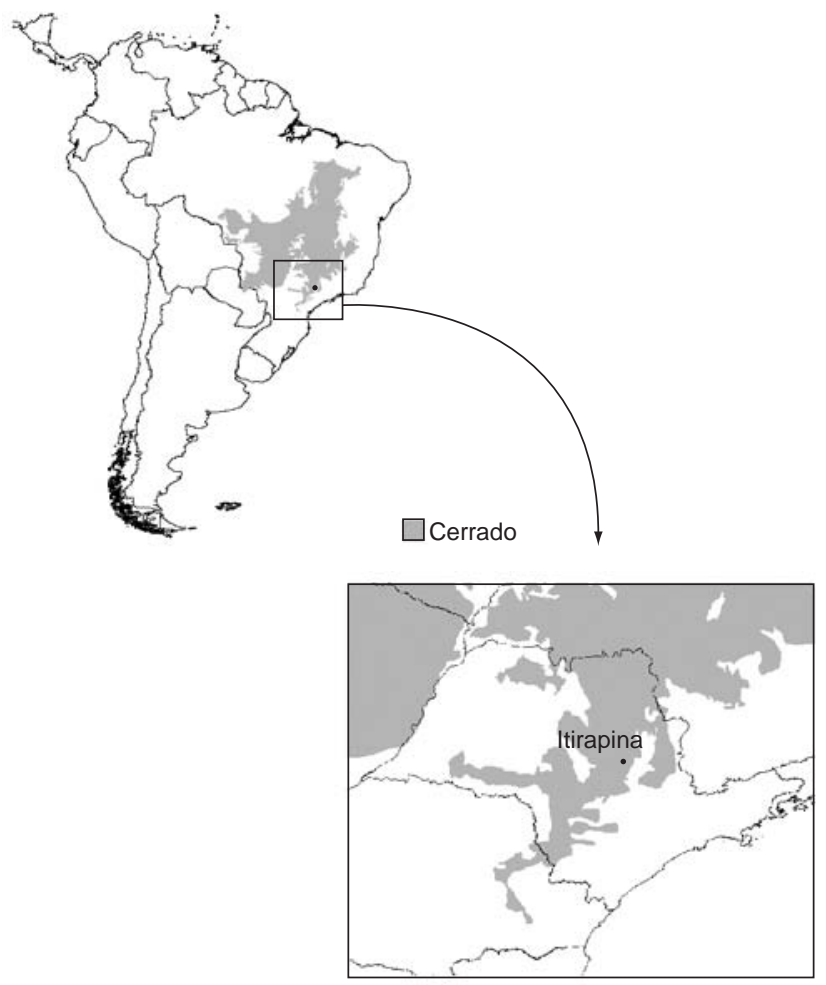

Figura 2. Localização da Estação Ecológica de Itirapina no domínio do Cerrado.

Figure 2. Location of the Estação Ecológica de Itirapina in the Cerrado domain. 
a março de 2000. Durante este período, as coletas tinham duração variável e ocorriam em intervalos esporádicos, totalizando 56 dias de campo. O período regular de amostragem foi de abril de 2000 até março de 2002, compreendendo quatro estações: as estações secas "1" (de abril a setembro de 2000) e "2" (de abril a setembro de 2001) e as estações chuvosas " 1 " (de outubro de 2000 a março de 2001) e "2" (de outubro de 2001 a março de 2002). Neste período, as amostragens foram quinzenais com duração de cinco dias cada, totalizando 240 dias de amostragem distribuídos em 48 viagens. As capturas de indivíduos foram feitas através de armadilhas de interceptação e queda (ver Cechin \& Martins 2000). Também foram feitas amostragens noturnas entre 4 e 8 de janeiro de 2005 .

As armadilhas de interceptação e queda foram distribuídas em três fisionomias de Cerrado: campo sujo, campo cerrado e nas bordas de duas matas de galeria. As armadilhas eram compostas por baldes de $100 \mathrm{~L}$ enterrados, interligados por cercas de tela plástica com $50 \mathrm{~cm}$ de altura, formando linhas de armadilhas. Cada linha tinha $45 \mathrm{~m}$ de comprimento e quatro baldes, um a cada quinze metros, intercalados pelas cercas-guia. As linhas foram dispostas em duplas, distantes em 100 m uma da outra, posicionadas em sobreposição a um eixo imaginário. Cada dupla de linhas foi considerada um ponto amostral, sendo instalados três pontos amostrais por fisionomia, com distância mínima de $500 \mathrm{~m}$. As armadilhas ficavam abertas durante os períodos de amostragem, permanecendo fechadas nos intervalos entre as coletas.

Para as amostragens noturnas, a EEI foi percorrida a partir do crepúsculo para identificação dos agregados reprodutivos. Os animais foram localizados pelas suas vocalizações. Para caracterização dos sítios de vocalização foram tomadas as seguintes medidas: profundidade da água, porcentagem de vegetação em um raio de $30 \mathrm{~cm}$ ao redor do macho (0-25, 26-50, 51-75 ou 76-100\%) e altura da vegetação. Também foram observados: grau de exposição do macho (exposto, parcialmente escondido, totalmente escondido), superfície de apoio ( solo nu, vegetação, água) e proporção do corpo imersa na água (corpo emerso, parcialmente imerso, totalmente imerso).

Os animais capturados tiveram registrado o comprimento rostrocloacal (CRC) (paquímetro Mitutoyo ${ }^{\circledR}$, precisão 0,5 mm) e a massa (dinamômetros Pesola, precisão 0,1 g). Para avaliação do clima foram coletados dados diários de temperaturas máxima e mínima, pluviosidade e umidade relativa do ar em uma estação metereológica situada a aproximadamente $10 \mathrm{Km}$ do local de estudo.

\section{Análises}

Para verificar o dimorfismo sexual foi aplicado teste de MannWhitney (Zar 1999) para a comparação do CRC, e feita análise de covariância (ANCOVA, Zar 1999) para comparação da massa (com a massa como variável dependente e o CRC como co-variável). Nestas análises foram incluídos todos os indivíduos amostrados cuja identificação do sexo foi possível, inclusive indivíduos capturados nos intervalos entre as coletas nas armadilhas fechadas. Consideramos adultos os machos com a região gular enegrecida e as fêmeas contendo óvulos em diferentes estágios de desenvolvimento, ou desovadas (com o oviduto hipertrofiado).

Para testar o uso do hábitat foram considerados animais capturados apenas durante o período regular de amostragem. Primeiramente foi testada a efetividade das réplicas através de análises de variância de Kruskal-Wallis (Zar 1999). Nesta análise, a abundância de cada ponto amostral registrada por coleta em uma mesma fisionomia foi considerada uma amostra. Em seguida foi testado se a abundância de indivíduos variou entre as fisionomias também com análises de variância de Kruskal-Wallis. Nesta análise a abundância de cada ponto amostral registrada por coleta por fisionomia foi considerada uma amostra.

Para analisar a abundância sazonal também foram considerados apenas os animais capturados durante o período regular de amostragem. Foi testado se a abundância variou entre as quatro estações amostradas através de análise de variância de Kruskal-Wallis. Em seguida foi investigado se a espécie apresentou preferência pelo começo, meio ou fim da estação chuvosa, comparando-se a abundância entre o primeiro, segundo e terceiro bimestre (outubro/ novembro, dezembro/janeiro e fevereiro/março), através de análise de variância de Kruskal-Wallis. Nestas análises, a abundância registrada em todas as fisionomias por coleta foi considerada uma amostra. Para verificar se existiu relação entre a abundância dos animais e as condições climáticas, foi utilizada uma análise de regressão múltipla incluindo as seguintes variáveis registradas por dia de coleta: abundância (como variável dependente), pluviosidade, temperatura mínima e máxima e umidade relativa do ar.

Todos os testes foram feitos com nível de significância de 0,05 no programa Statistica 6.0 (Statsoft 2001). Testes de Dunn a posteriori (Zar 1999) foram aplicados sempre que necessário no programa BioEstat 3.0 (Ayres et al. 1998).

\section{Resultados}

\section{Dimorfismo sexual}

Foram capturados 326 indivíduos: 213 nas armadilhas abertas (122 no período regular de amostragem e 91 no período de coletas preliminares), 93 nas armadilhas fechadas e 20 nas amostragens noturnas. Do total, foi possível determinar o sexo de 118 indivíduos: 54 machos e 64 fêmeas. As fêmeas foram significativamente maiores do que os machos $(\mathrm{U}=481,5 ; \mathrm{P}<0,01)$, mas a massa relativa não variou entre os sexos $\left(\mathrm{ANCOVA}_{1,115}=2,41 ; \mathrm{P}=0,12\right.$ ) (Tabela 1). Os machos apresentaram a região gular enegrecida, enquanto as fêmeas apresentaram esta região amarelada ou esbranquiçada. Não foram observadas outras diferenças no padrão de coloração entre os sexos.

\section{Sítio de vocalização}

$\mathrm{Na}$ EEI, os machos foram observados vocalizando principalmente em lagoas temporárias naturais formadas em campo sujo e campo limpo. Também foram observados machos vocalizando em outros ambientes onde havia vegetação encharcada, como nas valetas dos

Tabela 1. Valores médios e amplitudes de comprimento rostro-cloacal (CRC) e massa de machos e fêmeas de Elachistocleis cf. ovalis na Estação Ecológica de Itirapina \pm desvio padrão (DP).

Table 1. Mean values of snout-vent length (CRC) and weight of males and females of Elachistocleis cf. ovalis at the Estação Ecológica de Itirapina. DP, standard deviation.

\begin{tabular}{|c|c|c|c|c|c|c|}
\hline & \multicolumn{3}{|c|}{ Machos } & \multicolumn{3}{|c|}{ Fêmeas } \\
\hline & $\mathbf{N}$ & $\mathrm{X} \pm \mathrm{DP}$ & Amplitude & $\mathbf{N}$ & $\mathrm{X} \pm \mathrm{DP}$ & Amplitude \\
\hline CRC (mm) & 54 & $28,14+3,51$ & $16,3-36$ & 64 & $33,36+4,47$ & $19,4-42,7$ \\
\hline Massa (g) & 54 & $2,54+0,66$ & $1-3,9$ & 64 & $4,67+1,63$ & $1,3-8,5$ \\
\hline
\end{tabular}


aceiros por onde transitam os veículos na EEI e em outras áreas de vegetação antropizada nos arredores da estação (e.g. pastos). No entanto, os sítios de vocalização caracterizados estavam em ambiente não perturbado. O número de machos vocalizando foi maior nos dias chuvosos, no entanto esta relação não foi quantificada.

Os machos vocalizaram nas bordas das lagoas e em seu interior, em locais onde emergiam gramíneas formando touceiras de vegetação alagada (Figura 3a). Todos os machos observados $(\mathrm{N}=20)$ vocalizaram com o corpo escondido no interior de touceiras de gramíneas. Não foram observados machos vocalizando sobre o solo nu ou flutuando expostos. A posição dos machos foi sempre a mesma: o corpo ficava estendido e inclinado para cima (aprox. $45^{\circ}$ ), as mãos segurando a vegetação $(\mathrm{N}=20)$. A metade inferior do corpo permanecia sempre embaixo d'água, com as pernas apoiadas na vegetação submersa $(\mathrm{N}=20)$ (Figura 3b). Acima da água, a altura média da vegetação foi de 36,6 $\pm 15,64 \mathrm{~cm}$ (amplitude: 9 a $67 \mathrm{~cm}$; $\mathrm{N}=20$ ). A profundidade média do sítio de canto foi de 5,8 $\pm 4,11 \mathrm{~cm}$ (amplitude: 1 a $15,5 \mathrm{~cm} ; \mathrm{N}=20$ ). A porcentagem de vegetação ao redor da maioria dos machos $(\mathrm{N}=13)$ foi de 76 a $100 \%$, enquanto que para os machos restantes foi de 51 a $75 \%(\mathrm{~N}=4)$ e 25 a $50 \%(\mathrm{~N}=3)$.

\section{Uso de hábitat}

Considerando todos os indivíduos capturados no período regular de amostragem, aproximadamente $63,9 \%(\mathrm{~N}=78)$ foi capturado nas
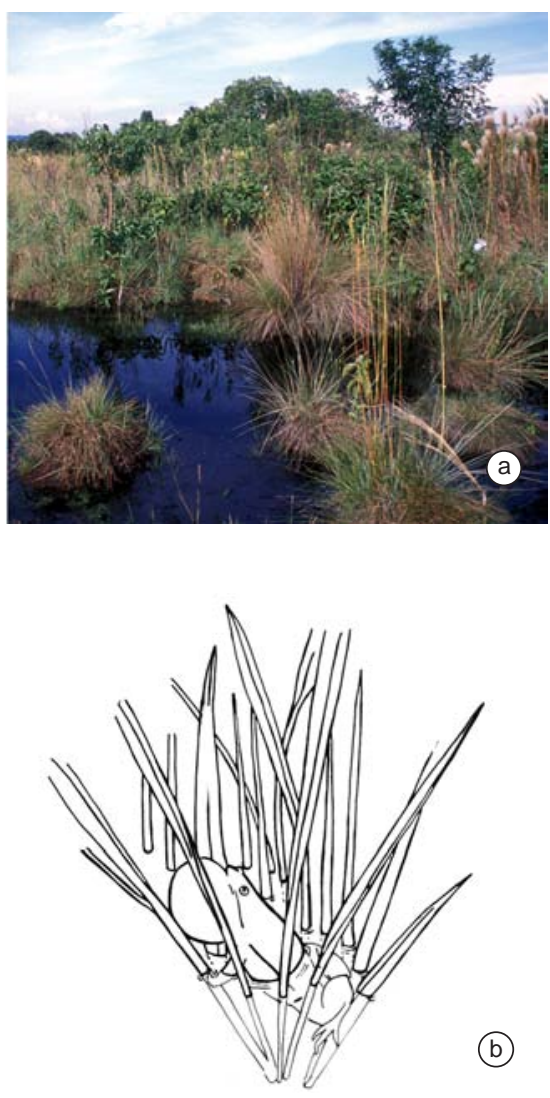

Figura 3. Sítio e posição de vocalização de Elachistocleis cf. ovalis na Estação Ecológica de Itirapina. a) margem e touceiras de gramíneas alagadas em uma lagoa temporária; e b) ilustração da postura típica de Elachistocleis cf. ovalis vocalizando.

Figure 3. Vocalization site and body posture of Elachistocleis cf. ovalis at the Estação Ecológica de Itirapina. A: edge and flooded grasses agglomerates in a temporary pond; and B: ilustration of typical posture of Elachistocleis cf. ovalis vocalizing. armadilhas da borda de mata de galeria, 30,3\% ( $\mathrm{N}=37)$ no campo sujo e apenas $5,7 \%(\mathrm{~N}=7)$ em campo cerrado (Figura 4). A abundância de indivíduos variou significativamente entre as fisionomias $(\mathrm{H}=16,6 ; \mathrm{P}<0,01, \mathrm{~N}=432)$. Os testes a posteriori indicaram diferença significativa entre campo sujo e borda de mata de galeria e entre o campo cerrado e borda de mata de galeria (Tabela 2). Não foram encontradas diferenças de abundância entre réplicas (campo sujo: $\mathrm{H}=0,2 ; \mathrm{P}=0,9 ; \mathrm{N}=144$; campo cerrado: $\mathrm{H}=0,04 ; \mathrm{P}=1$, $\mathrm{N}=144$; borda de mata de galeria: $\mathrm{H}=0,04 ; \mathrm{P}=1, \mathrm{~N}=144$ ).

\section{Abundância sazonal}

Foram registradas 108 capturas durante as estações chuvosas e apenas 14 durante as estações secas. A variação de abundância entre as quatro estações amostradas foi significativa $(\mathrm{H}=22,5, \mathrm{P}=<<0,01$, $\mathrm{N}=48$ ). Testes a posteriori indicaram diferença significativa entre estações secas e chuvosas (com exceção das estações seca 1 e chuvosa 2), mas não entre estações semelhantes (Tabela 3). Também não foi encontrada variação de abundância entre os bimestres da estação chuvosa $(\mathrm{H}=8,82, \mathrm{P}=0,12, \mathrm{~N}=24)$, mas as capturas tiveram um pico nos meses de novembro (primeira estação chuvosa) e dezembro (segunda estação) (Figura 5).

A regressão linear múltipla $\left(\mathrm{R}^{2}=0,11, \mathrm{~F}_{(4,235)}=7,79, \mathrm{P}<0,01\right)$ indicou que a abundância de indivíduos capturados foi influenciada de forma significativa pela umidade relativa do ar $\left(\mathrm{R}^{2} \mathrm{p}=0,56, \mathrm{P}<0,01\right)$ e pela temperatura máxima $\left(\mathrm{R}^{2} \mathrm{p}=0,43, \mathrm{P}=0,04\right)$ (Figura 5), mas não pela pluviosidade $\left(R^{2} \mathrm{p}=0,12, \mathrm{P}=0,24\right)$ ou temperatura mínima $\left(\mathrm{R}^{2} \mathrm{p}=57, \mathrm{P}=0,45\right)$.

A presença de fêmeas reprodutivas, contendo óvulos maduros, não foi concentrada em nenhum mês específico, sendo que das sete registradas, duas foram capturadas na estação seca (agosto e setembro) e as outras cinco durante estações chuvosas (outubro, novembro, dezembro, janeiro e março).

\section{Discussão}

O dimorfismo sexual em tamanho já havia sido observado em populações de Elachistocleis ovalis do Rio Grande do Sul (Kwet \& Di Bernardo 1999) e na Serra do Cipó, Minas Gerais (Eterovick \& Sazima 2004). Também foi encontrado dimorfismo semelhante em E. bicolor (Rodrigues et al. 2003), em E. erythrogaster (Kwet \& Di Bernardo 1999) e em E. skotogaster (Lavilla et al. 2003). Dimorfismo

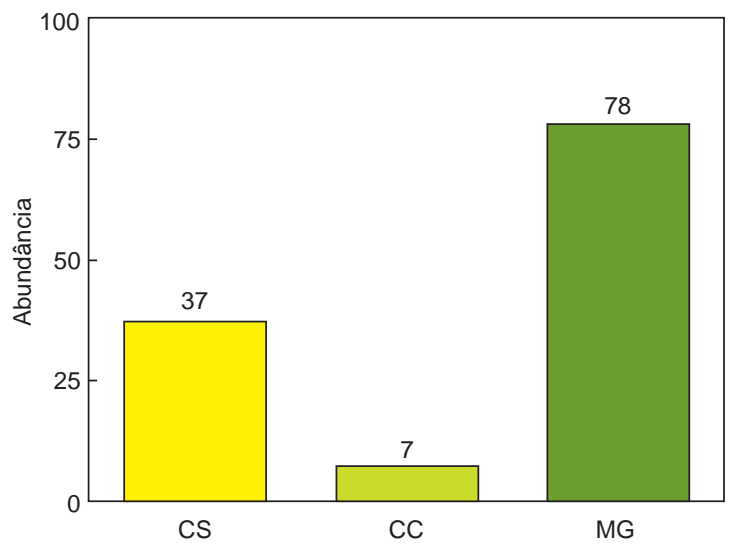

Figura 4. Uso do hábitat por Elachistocleis cf. ovalis na Estação Ecológica de Itirapina. CS: campo sujo; CC: campo cerrado; e MG: borda de mata de galeria.

Figure 4. Habitat use by Elachistocleis cf. ovalis at the Estação Ecológica de Itirapina. CS: campo sujo; CC: campo cerrado; and MG: gallery forest edge. 
Tabela 2. Testes a posteriori comparando a abundância de Elachistocleis cf. ovalis entre diferentes fisionomias na Estação Ecológica de Itirapina. ns = não significativo

Table 2. A posteriori tests comparing abundance of Elachistocleis cf. ovalis among different physiognomies at the Estação Ecológica de Itirapina. ns, non significant.

\begin{tabular}{lcc}
\hline Hábitats comparados & z calculado & z crítico \\
\hline Campo sujo e campo cerrado & 1,53 & 2,39 \\
Campo sujo e borda de mata de galeria & 2,51 & 2,39 \\
Campo cerrado e borda de mata de galeria & $<0,05$ & 2,39 \\
\hline
\end{tabular}

Tabela 3. Testes a posteriori comparando a abundância de Elachistocleis cf. ovalis em estações secas e chuvosas na Estação Ecológica de Itirapina. ns= não significativo; seca 1 = abril a setembro de 2000; seca 2 = abril a setembro de 2001 ; chuvosa $1=$ outubro de 2000 a março de 2001 e chuvosa 2 = outubro de 2001 a março de 2002.

Table 3. A posteriori tests comparing abundance of Elachistocleis cf. ovalis in dry and wet seasons at the Estação Ecológica de Itirapina. ns, non significant; dry 1, April to September 2000; dry 2, April to September 2001; wet 1, October 2000 to march 2001 and wet 2, October 2001 to march 2002.

\begin{tabular}{|c|c|c|c|}
\hline Estações comparadas & $\mathrm{z}$ calculado & z crítico & $\mathbf{P}$ \\
\hline seca 1 e seca 2 & 0,85 & 2,64 & ns \\
\hline seca 1 e chuvosa 1 & 3,33 & 2,64 & $<0,05$ \\
\hline seca 1 e chuvosa 2 & 2,16 & 2,64 & ns \\
\hline seca 2 e chuvosa 1 & 4,18 & 2,64 & $<0,05$ \\
\hline seca 2 e chuvosa 2 & 3,01 & 2,64 & $<0,05$ \\
\hline chuvosa 1 e chuvosa 2 & 1,17 & 2,64 & ns \\
\hline
\end{tabular}

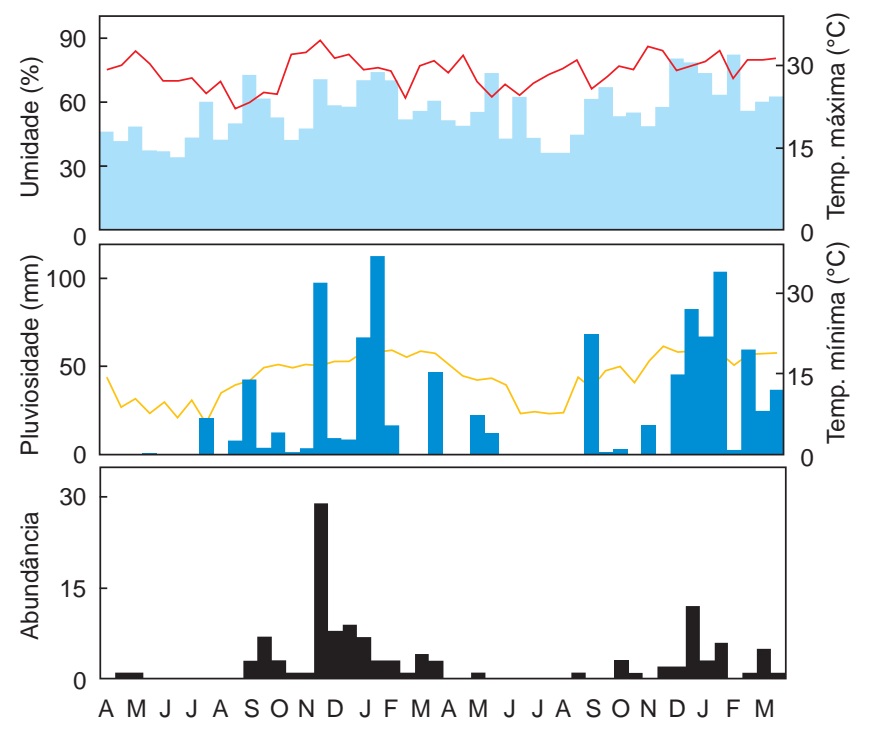

Figura 5. Umidade relativa do ar, pluviosidade, temperaturas mínima e máxima e abundância de Elachistocleis cf. ovalis na Estação Ecológica de Itirapina entre abril de 2000 e março de 2002.

Figure 5. Relative air humidity, rainfall, maximum and minimal temperatures and abundance of Elachistocleis cf. ovalis at the Estação ecológica de Itirapina from April 2000 to March 2002.

sexual com fêmeas maiores do que machos é comum entre anuros (Shine 1979), mas interpretações de como esta característica surgiu são variadas. Fêmeas maiores seriam selecionadas por produzirem óvulos maiores e/ ou em maior número (Crump \& Kaplan 1979, Prado et al. 2000). Por sua vez, machos não atingiriam tamanhos grandes devido a restrições energéticas relacionadas à reprodução (Woolbright 1983), ou à maior pressão de predação decorrente de atividades relacionadas (Ryan 1985). Em E. cf. ovalis ou mesmo em outras espécies do gênero as causas do dimorfismo podem estar mais relacionadas ao aumento de fecundidade das fêmeas, pois o padrão de reprodução explosivo pode aliviar a pressão sobre os machos por ocorrer durante um período mais curto se comparado ao padrão de reprodução prolongado (Sebbins \& Cohen 1995).

Embora pouco detalhadas, as descrições dos sítios de canto e da posição de vocalização de E. ovalis na literatura são semelhantes ao registrado para a população da EEI. Machos de E. ovalis foram registrados vocalizando em água rasa entre gramíneas com o corpo inclinado, parcialmente imerso (Kwet \& Di Bernardo 1999, Achaval \& Olmos 2003, Eterovick \& Sazima 2004). Outros registros para o gênero incluem E. bicolor (Rodrigues et al, 2003), E. erythrogaster (Kwet \& Di Bernardo 1999) e E. skotogaster (Lavilla et al. 2003) vocalizando em ambientes e posições semelhantes.

Comportamento semelhante em espécies relacionadas é um indicativo de restrições filogenéticas (Brooks \& McLennan 1991), no entanto a semelhança no sítio e posição de vocalização pode ser causada pelas condições do ambiente e não por razões históricas. A falta de complexidade estrutural dos ambientes abertos parece ser limitante na diferenciação dos sítios de vocalização, principalmente para os anuros que vocalizam no chão ou na água (Rossa-Feres \& Jim 2001). Como consequiência, o número de espécies reproduzindo nestes ambientes freqüentemente é maior do que o número de microhábitats disponíveis (Cardoso et al. 1989). Elachistocleis erythrogaster (Kwet \& Di Bernardo 1999) e E. skotogaster (Lavilla et al. 2003), e mesmo E. cf ovalis (presente estudo), foram registrados também vocalizando em ambiente alterado, o que sugere que o grupo apresenta poucas exigências de hábitat, e que provavelmente compartilha grande plasticidade fenotípica em relação ao sítio de vocalização. Entretanto, a semelhança que determinados ambientes antropizados (p. e. pastagens) mantém com os ambientes abertos em geral pode facilitar sua ocupação por estas espécies.

As bordas das mata de galeria foram os ambientes mais utilizados por E. cf. ovalis na EEI. Citações do uso de hábitat por espécies de Elachistocleis baseadas no mesmo método de coleta são raras. Em um único estudo encontrado, Elachistocleis sp. foi registrado tanto em formações abertas quanto florestadas (Strussmann 2000). No Pantanal, indivíduos de E. cf. bicolor foram observados dentro dos capões de mata durante a estação seca, utilizando corpos d'água temporários apenas na época reprodutiva (C. P. A. Prado, dados não publicados). Outros estudos indicam que as espécies do gênero são 
restritas a formações abertas (Kwet \& Di Bernardo 1999, Jim 2003, Brandão \& Araújo 2001), mas estes resultados podem ser enviesados pelo método de coleta utilizado nestes outros estudos, baseado diretamente na observação da atividade reprodutiva. Todas as espécies do gênero de que se têm registros na literatura utilizam ambientes abertos para reprodução (Kwet \& Di Bernardo 1999, Achaval \& Olmos 2003, Rodrigues et al. 2003, Lavilla et al. 2003, Eterovick \& Sazima 2004).

O padrão de abundância nas fisionomias pode também estar relacionado à proximidade destes ambientes com os brejos onde se formam os agregados reprodutivos, já que o campo cerrado é a fisionomia mais distante e também a menos populosa (Brasileiro et al. 2005). Durante a estação chuvosa, os animais podem utilizar as outras fisionomias como abrigo (especialmente a borda de mata de galeria), retornando aos corpos d'água diariamente, e ter sido capturados em trânsito nestes trajetos.

Elachistocleis cf. ovalis apresentou abundância sazonal, com maior atividade durante a estação chuvosa. Esta estação parece coincidir com o período reprodutivo de outras populações (Rossa-Feres \& Jim 1994, Kwet \& Di Bernardo 1999, Achaval \& Olmos 2003, Eterovick \& Sazima 2004, Toledo et al. 2003) e outras espécies do gênero (Strussmann 2000, Rodrigues et al. 2003). Provavelmente o aumento no número de indivíduos capturados resulta da maior atividade reprodutiva na estação chuvosa. Entretanto não houve diferença de abundância entre os bimestres da estação chuvosa, indicando que $E$. cf. ovalis pode se reproduzir ao longo de todo o período na EEI.

A atividade exclusiva durante a estação chuvosa é comum entre espécies que se reproduzem em ambiente com vegetação aberta (Rossa-Feres \& Jim 1994), constituindo também um padrão esperado para os anuros que habitam o Cerrado (Colli et al. 2002, Prado et al. 2005). Alguns registros indicam que E. ovalis permanece a maior parte do ano enterrado, só saindo após chuvas fortes para se reproduzir (Barrio-Amorós 2004, Eterovick \& Sazima 2004), outra estratégia comum para o grupo no bioma (Colli et al. 2002). Estes dois comportamentos já foram observados em outro microhilídeo neotropical que ocorre no Cerrado, Dermatonotus muelleri (Nomura 2003).

O padrão sazonal de abundância de Elachistocleis $c f$. ovalis na EEI pode ser explicado pelo aumento da umidade relativa do ar e da temperatura máxima, duas variáveis climáticas cuja influência sobre a atividade dos anuros já foi documentada. No Cerrado, foi registrado que a umidade relativa do ar e a temperatura média influenciaram o número de machos vocalizando ao longo do ano em uma população de Physalaemus centralis, localizada a poucos quilômetros da EEI (Brasileiro \& Martins 2006). De fato, o aumento de temperatura parece afetar o número de espécies em atividade reprodutiva em locais com sazonalidade climática acentuada (Vasconcelos \& Rossa-Feres 2005, Prado et al. 2005). Dentre os microhylideos, Dermatonotus muelleri teve abundância mensal positivamente correlacionada com a chuva e temperatura mínima do ar (Nomura 2003), enquanto que duas outras espécies australianas tiveram o número de machos vocalizantes ao longo do dia influenciado pela umidade relativa do ar (Hauselberger \& Alford 2005). Possivelmente, outras variáveis não tomadas influenciam a atividade sazonal (e provavelmente diária) de E. cf. ovalis no Cerrado. Em Physalaemus centralis a atividade reprodutiva ao longo do ano parece ser precedida também por variação na pressão barométrica (Brasileiro \& Martins 2006). Em regiões temperadas, com sazonalidade climática, a atividade reprodutiva dos anfíbios pode ser regulada também pelo fotoperíodo (Sebbins \& Cohen 1995).

Embora este estudo tenha sido realizado em um prazo de dois anos, não houve flutuação significativa na abundância de um ano para o outro. Em um estudo realizado na Serra do Cipó foi notada a ausência desta espécie em relação a coletas anteriores, mas devido ao caráter de sua reprodução a sugestão de que a população estivesse declinando seria pouco fundamentada (Eterovick et al. 2005). Informações sobre declínios populacionais de anuros brasileiros são ainda escassas (Silvano \& Segalla 2005) e os estudos sobre o tema ainda estão em sua fase inicial. A escassez de informação é o principal obstáculo na determinação do estado de conservação dos anuros brasileiros (Pimenta et al. 2005). No caso de E. ovalis e da maioria das espécies de distribuição ampla a determinação segura do status de conservação é extremamente limitada pela ausência de estudos taxonômicos abrangentes.

\section{Agradecimentos}

Nós agradecemos ao Instituto Florestal pelo acesso à Estação Ecológica de Itirapina e especialmente a Denise Zanchetta pelo suporte logístico. Agradecemos ao Ibama pela licença de coleta (02027.010426/99-21), a Ripasa S.A. pelo fornecimento dos dados climáticos e a Alexandro Tozetti pela foto do espécime. Agradecemos à Cynthia P. de A. Prado, Hilton M. Oyamaguchi e Elaine M. Lucas pelas contribuições ao manuscrito. Agradecemos também a todos os que participaram nos trabalhos de campo, especialmente Ricardo J. Sawaya, Mara C. Kiefer, Graziella Giraldelli, Felipe Spina, Vagner Ariedi Jr, Glauco Machado, Vinicius Bonato e Tiago M. Castellar. Ao Marcio Martins pelo apoio logístico e coordenação do projeto "Ecologia dos Cerrados de Itirapina. Agradecemos ao Programa de Pós Graduação em Ecologia, Instituto de Biociências-USP; à FAPESP, $\mathrm{CNPq}$ e Neotropical Grassland Conservancy pelo apoio financeiro $\mathrm{CAB}$ agradece à FAPESP pela bolsa de Doutorado; MTCT agradece à CAPES pela bolsa de mestrado. Esta é a publicação número 29 do projeto "Ecologia dos Cerrados de Itirapina".

\section{Referências Bibliográficas}

ACHAVAL, F. \& OLMOS, A. 2003. Anfibios y reptiles del Uruguay. $2^{\mathrm{a}}$ edición corregida y aumentada. Graphis, Impresora, Montevideo, Uruguay.

AYRES, M., AYRES Jr., M.A., AYRES, D.L. \& DOS SANTOS, A.S. 1998. BioEstat: aplicações estatísticas nas áreas das ciências biológicas e médicas. Publicações Avulsas Mamirauá. 193p. Acompanha CD-ROM para PC compatível com Windows 95. Manaus: Sociedade Civil Mamirauá.

BARRIO-AMORÓS, C.L. 2004. Herpetological approach to the Venezuelan Llanos. Arassari and Andigena technical report 1.www.arassari.com / www.andigena.org

BRANDÃO, R.A. \& ARAÚJO, A.F.B. 2001. A herpetofauna associada às matas de galeria no Distrito Federal. In Cerrado: caracterização e recuperação das matas de galeria (J.F Ribeiro, C. E. L. Fonseca \& J.C. Sousa-Silva, eds.) Embrapa, Planaltina. p.561-606.

BRASILEIRO, C.A. \& MARTINS, M.R.C. 2006. Breeding biology of Physalaemus centralis Bokermann, 1962 (Anura: Leptodactylidae) in southeastern Brazil. J. Nat. Hist. 40:1199-1209.

BRASILEIRO, C.A.; SAWAYA, R.J., KIEFER, M.C. \& MARTINS, M. 2005. Amphibians of an open Cerrado fragment in Southeastern Brazil. Biota Neotrop. 5(2): http://www.biotaneotropica.org.br/v5n2/pt/abstract?artic le+BN00405022005

BROOKS, D.R. \& MC LENNAN, D.A. 1991. Phylogeny, ecology and behavior: A research program in comparative biology. The University of Chicago Press, Chicago and London.

CARDOSO, A.J., ANDRADE, G.V. \& HADDAD, C.F.B. 1989. Distribuição espacial em comunidades de anfíbios (Anura) no sudeste do Brasil. Rev. Bras. Biol. 49:241-249.

CAVALCANTI, R.B. \& JOLY, C.A. 2002. Biodiversity and conservation priorities in the Cerrados. In The Cerrados of Brazil: Ecology and Natural History of a Neotropical Savanna (Oliveira, P.S. \& Marquis, R.J. eds.). Columbia University Press, Columbia. p.351-367. 
CECHIN, S.Z. \& MARTINS, M. 2000. Eficiência de armadilhas de queda (pitfall traps) em amostragens de anfíbios e répteis no Brasil. Revta. Bras. Zool. 17:729-740.

COLLI, G.R.; BASTOS, R.P. \& ARAUJO F.B. 2002. The character and dynamics of the Cerrado herpetofauna. In The Cerrados of Brazil: Ecology and Natural History of a Neotropical Savanna (P.S Oliveira \& R. J. Marquis, eds.). Columbia University Press, Columbia. p.223-239.

CRUMP, M.L. \& KAPLAN, R.H. 1979. Cluch energy portioning of tropical tree frogs (hylidae). Copeia 1979: 626-635.

ETEROVICK, P.C. \& SAZIMA, I. 2004. Anfíbios da Serra do Cipó - Minas Gerais - Brasil. Amphibians from the Serra do Cipó. PUC Minas, Belo Horizonte.

ETEROVICK, P.C., CARNAVAL, A.C.O.Q., BORGES-NOJOSA, D.M. SILVANO, D.L., SEGALLA, M.V. \& SAZIMA, I. 2005. Amphibian declines in Brazil: an overview. Biotropica 37(2):166-179.

FROST, D.R. 2004. Amphibian Species of the World: an Online Reference. Version 3.0 (22 August, 2004). Electronic Database accessible at http:// research.amnh.org/herpetology/amphibia/index.html. American Museum of Natural History, New York, USA.

HAUSELBERGER, K.F. \& ALFORD, R.A. 2005. Effects of season and weather on calling in the Australian microhylid frogs Austrochaperina robusta and Cophixalus ornatus. Herpetologica 61(4):349-363.

JIM, J. 2003. Aspectos gerais da anurofauna da região de Botucatu. Em: Flora e fauna: um dossiê ambiental (W. Uieda \& L. M. Paleari, org.). Editora Unesp, São Paulo. p.75-90.

KWET, A \& DI-BERNARDO, M. 1998. Elachistocleis erythrogaster, a new Microhylid species from Rio Grande do Sul, Brazil. Stud. Neotrop Fauna \& Environm. 33: 7-18.

KWET, A \& DI-BERNARDO, M. 1999. Anfíbios. Amphibien. Amphibians. EDIPUCRS, Porto Alegre.

LAVILLA, E.O., VAIRA, M. \& FERRARI, L. 2003. A new species of Elachistocleis (Anura: Microhylidae) from the Andean Yungas of Argentina, with comments on the Elachistocleis ovalis-E. bicolor controversy. Amphibia-Reptilia 24: 269-284.

MANTOVANI, J.E. \& PEREIRA, A. 1998. Estimativa da integridade da cobertura vegetal do Cerrado/Pantanal através de dados TM/LANDSAT. In Workshop Ações Prioritárias para Conservação da Biodiversidade do Cerrado e Pantanal. Ministério do Meio Ambiente, FUNATURA, Conservation International, Fundação Biodiversitas, Universidade de Brasília. Web: www.bdt.org.br/workshop/cerrado/br

NOMURA, F. 2003. Ecologia reprodutiva e comportamento de forrageio e escavação de Dermatonotus muelleri (Boettger, 1885) (Anura, Microhylidae). Dissertação de mestrado, Unesp, São José do Rio Preto.

PIMENTA, B.V.S., HADDAD, C.F.B., NASCIMENTO, L.B., CRUZ, C.A.G. \& POMBAL Jr., J.P. 2005. Comment on "Status and Trends of Amphibian Declines and Extinctions Worldwide". Science 309.

PRADO, C.P.A., UETANABARO, M. \& LOPES, F.S. 2000. Reproductive strategies of Leptodactylus chaquensis and L. podicipinus in the Pantanal, Brasil. J. Herpetol. 34(1):135-139.

PRADO, C. P. A., UETANABARO, M. \& HADDAD, C. F. B. 2005. Breeding activity patterns, reproductive modes, and habitat use by anurans (Amphibia) in a seasonal environment in the Pantanal, Brazil. AmphibiaReptilia 26: 211-221.

RODRIGUES, D. J., LOPES, F. S. \& UETANABARO, M. 2003. Padrão reprodutivo de Elachistocleis bicolor (Anura, Microhylidae) na Serra da Bodoquena, Mato Grosso do Sul, Brasil. Iheringia, Sér. Zool. 93(4):365371.

ROSSA-FERES, D. C. \& JIM, J. 1994. Distribuição sazonal em comunidades de anfíbios anuros na região de Botucatu, São Paulo. Rev. Bras. Biol. 54(2): 323-334.
ROSSA-FERES, D.C. \& JIM, J. 2001. Similaridade do sítio de vocalização em uma comunidade de anfíbios anuros na região noroeste do estado de São Paulo, Brasil. Rev. Bras. Biol. 18(2):439-454.

ROSSA-FERES, D.C., JIM, J. \& FONSECA, M.G. 2004. Diets of tadpoles from a temporary pond in southeastern Brazil (Amphibia, Anura). Rev. Bras. Zool. 21(4):745-754

RYAN, M.J. 1985. The Tungara frog: a study in sexual selection and communication. The University of Chicago Press, Chicago.

SEBBINS, R.C. \& COHEN, N.W. 1995. A natural history of amphibians. Princeton University Press, New Jersey.

SILVANO, D.L. \& SEGALLA, M.V. 2005. Conservation of Brazilian amphibians. Conservation Biology 19(3):653-658.

SHINE, R. 1979. Sexual selection and sexual dimorphism in the Amphibia. Copeia 2: 297-306

SOLÉ, M., KETTERL, J., DI-BERDARDO, M. \& KWET, A. 2002. Ants and termites are the diet of the microhylid frog Elachistocleis ovalis (Schneider, 1799) at an Arauraria forest in Rio Grande do Sul, Brazil. Herpetol. Bull. 79:14-17.

STATSOFT, INC. 2001. STATISTICA (data analysis software system), version 6. www.statsoft.com.

STRUSMANN, M. 2000. Capítulo IV: Herpetofauna. In Fauna silvestre da região do Rio Manso, MT. (C. R. J. Alho, org.). Brasília. p.153-189.

TOLEDO, L.F., ZINA, J. \& HADDAD, C.F.B. 2003. Distribuição espacial e temporal de uma comunidade de anfíbios anuros do município de Rio Claro, São Paulo, Brasil. Holos Environm. 3 (2): 136-149.

VASCONCELOS, T.S. \& ROSSA-FERES, D.C. 2005. Diversidade, distribuição espacial e temporal de anfíbios anuros (Amphibia, Anura) na região noroeste do estado de São Paulo, Brasil. Biota Neotrop. 5(2): http:// www.biotaneotropica.org.br/v5n2/pt/abstract?article+BN01705022005

VICENTE, L.E., SOUZA FILHO, C. R. \& PEREZ FILHO, A. 2005. Mapeamento de formações arenosas em fragmentos de Cerrado utilizando dados e produtos do sensor ASTER. In XII Simpósio Brasileiro de Sensoriamento Remoto. INPE, Goiânia, p.3419-3426.

WELLS, K.D. 1977. The courtship of frogs. In The reproductive biology of amphibians. (D. H. Taylor \& S. I. Guttman, eds.). Plenum, New York. p.475.

WOOLBRIGHT, L.L. 1983. Sexual selection and size dimorphism in anuran amphibia. Am. Nat. 121(1):110-119.

ZAR, J.H.1999. Biostatistical Analysis. Third ed. Prentice-Hall.

Título: Dimorfismo sexual, uso do ambiente e abundância sazonal de Elachistocleis cf. ovalis (Anura: Microhylidae) em um remanescente de Cerrado no estado de São Paulo, sudeste do Brasil.

Autores: Thomé MTC. e Brasileiro CA.

Biota Neotropica, Vol.7 (número 1): 2006

http://www.biotaneotropica.org.br/v7n1/pt/abstract?article+ bn00307012007

Recebido em 15/08/06 - Versão Reformulada recebida em 10/11/06 - Publicado em 01/01/07

ISSN 1676-0603 